\title{
MASSLESS COMPOSITES WITH MASSIVE CONSTITUENTS
}

\author{
Savas DIMOPOULOS ${ }^{1.2}$ \\ Institute of Theoretical Physics, Stanford University, Stanford, California 94305 , \\ Institute of Theoretical Physics, University of California, Santa Barbara, Califomia 93106 \\ and \\ Department of Physics, University of Michigan, Ann Arbor, Michigan 48109, USA \\ John PRESKILL ${ }^{3}$ \\ Lyman Laboratory of Physics, Harvard University, Cambridge, Massachusetts 02138, USA
}

Received 29 October 1981

We construct model field theories in which a confining gauge interaction binds massive elementary fermions into massiess composite particles. The massless composites are either Goldstone bosons or spin- $-\frac{1}{2}$ fermions. In these models, the manner in which exact chiral symmetries are realized changes at a critical value of the elementary fermion mass of order $\left(e^{2} / 16 \pi^{2}\right) \Lambda$, where $A$ is the confinement scale and $e$ is a weak gauge coupling.

\section{Introduction}

Seeking models of the known elementary particle interactions which are completely "natural" and in which quark and lepton masses are calculable, many authors have studied gauge theories without elementary scalar fields or bare fermion masses [1-9]. A realistic theory of this type requires new strong interactions which respect large (approximate) flavor symmetry groups. Hence, constructors of such theories face a central theoretical question: How does a given asymptotically free gauge theory choose to realize its global flavor symmetries?

This question has two parts. (1) What subgroup of the flavor group escapes dynamical spontaneous symmetry breakdown? (2) What fermion multiplets are kept massless by the unbroken flavor symmetry?

Clearly it is essential to answer these questions in order to determine the phenomenological consequences of a proposed model. Corresponding to each spontaneously broken generator of the flavor group is a composite Goldstone boson. In a realistic theory, this Goldstone boson may be eaten by a gauge boson, may remain exactly massless, or may become a massive pseudo-Goldstone boson

' Address after January 1, 1982: Lyman Laboratory of Physics, Harvard University, Cambridge, Massachusetts 02138.

${ }_{2}^{2}$ Research supported in part by the National Science Foundation under grant no. PHY77-27084.

${ }^{3}$ Research supported in part by the National Science Foundation under grant no. PHY77-22864, and by the Harvard Society of Fellows. 
due to weak flavor-symmetry-breaking perturbations. The pseudo-Goldstone boson spectrum is one of the interesting predictions of a theory without elementary scalar fields $[1,4]$.

The unbroken flavor symmetry may require that massless fermions occur in the spectrum of the theory [9]; if the gauge interaction is confining, these fermions must be composite states. They are candidates for quarks and leptons, and, like the pseudo-Goldstone bosons, they can acquire small calculable masses from flavor-symmetry-breaking perturbations $[7,8]$.

Determining how flavor symmetries are realized in gauge theories is a difficult strong-coupling problem for which the general solution is not yet known. However, progress has recently been made. In ref. [9],' $t$ Hooft proposed an algebraic condition that must be satisfied by the massless composite fermions in a confining gauge theory; namely, that the massless composite fermions and elementary fermions produce equal Adler-Bell-Jackiw anomalies for the unbroken flavor group ${ }^{\star}$. Using this algebraic condition and a subsidiary condition to be described below, 't Hooft concluded that the $\mathrm{SU}(n)_{\mathrm{L}} \times \mathrm{SU}(n)_{\mathrm{R}} \times \mathrm{U}(1)_{\mathrm{V}}$ flavor symmetry of $\mathrm{QCD}$, with 3 colors and $n$ massless flavors must be spontaneously broken for $n>2$.

The subsidiary condition used by ' $t$ Hooft was elaborated in ref. [11], where it was called the "persistent-mass condition" (PMC). In brief, this condition requires that a composite particle cannot remain exactly massless while the mass of one of its constituents varies over a finite range of values. The PMC and its consequences will be discussed in more detail in sect. 2 .

The PMC actually leads to a stronger conclusion than that stated by ' $t$ Hooft. We say that a flavor symmetry group is non-chiral if it allows masses for all the elementary fermions which transform non-trivially under the group. The PMC implies that a continuous non-chiral flavor symmetry cannot be spontaneously broken [11]. Combining this result with ' $t$ Hooft's analysis, we can conclude that the $\mathrm{SU}(n)_{\mathrm{L}} \times \mathrm{SU}(n)_{\mathrm{R}} \times \mathrm{U}(1)_{\vee}$ flavor symmetry of $\mathrm{QCD}$ with $n$ massless flavors must be spontaneously broken to $\mathrm{SU}(n)_{V} \times \mathrm{U}(1)_{V}$ if $n>2$. (For $n=2$ completely unbroken $S U(2)_{L} \times S U(2)_{R} \times U(1)_{V}$ cannot be excluded by 't Hooft's arguments.)

But is the PMC true? It appears to be based on intuitive notions about bound states rather than general principles of quantum field theory. In ref. [11] a nonrenormalizable model was described, which, in an untrustworthy approximation, appeared to violate the PMC. That model had an exactly massless Goldstone boson which was a bound state of massive elementary fermions.

Our main purpose in this paper is to present two more examples of models in which the PMC fails. Both models are renormalizable quantum field theories, and hence demonstrate that the non-renormalizability of the model in ref. [11] was not essential. Our first example contains a massless Goldstone boson, the second a

\footnotetext{
* The justification of this condition is further discussed in ref. [10].
} 
massless composite fermion; the second example dispels the notion that the PMC may be correct when applied to composite fermions, but not for composite bosons.

The crucial feature of both examples is a weak gauge interaction which competes with the intrinsic mass of an elementary fermion to determine the alignment of the vacuum $[1,12]$. We will compute and minimize an effective potential to show that the symmetry of the vacuum changes at a critical value of the elementary fermion mass of order $\left(e^{2} / 16 \pi^{2}\right) \Lambda$, where $e$ is the weak gauge coupling and $\Lambda$ is the confinement scale of the strong interaction responsible for the binding. Massless composites with massive constituents occur only if the elementary fermion mass is smaller than this critical value.

This paper is organized as follows: In sect. 2, the PMC is discussed, and the conclusions of ref. [11] are reviewed. The importance of vacuum alignment in determining the massless spectrum of a theory is illustrated by an example in sect. 3. In sects. 4 and 5 , we present our models containing massless composite particles with massive constituents; we describe a composite boson in sect. 4 , and a composite fermion in sect. 5. Details concerning the analysis of these models are discussed in two appendices. Sect. 6 contains our conclusions.

\section{The persistent-mass condition}

To demonstrate the PMC, we will consider, for definiteness, $n$-flavor QCD. If all $n$ flavors are massless, this theory is invariant under the flavor group $\mathrm{G}_{\mathrm{f}}=$ $S U(n)_{L} \times S U(n)_{R} \times U(1)_{V}$. Because the elementary fermions, the quarks, have $G_{f}$ anomalies, the spectrum of QCD must contain massless fermions, unless $G_{f}$ is spontaneously broken [9]. If quarks are confined, these massless fermions must be color-singlet composite states. Thus, there are surely massless composite particles in QCD; either Goldstone bosons, or massless composite fermions, or both.

Now suppose that one quark has an intrinsic mass $m$, while the other $(n-1)$ quarks remain massless. Let $M$ be the mass of a composite particle, massless in the $\mathrm{G}_{\mathrm{f}}$ symmetry limit, which contains the massive quark. How will $M$ behave as a function of $m$ ?

By assumption, $M=0$, when $m=0$. We also know how $M$ behaves as $m \rightarrow \infty$. By the Appelquist-Carazzone theorem [13], the massive quark must decouple as $m \rightarrow \infty$, and any bound state containing it must also become infinitely massive.

In fig. 1 are shown three possible types of behavior of $M$ as a function of $m$. In fig. $1 \mathrm{a} M$ is non-vanishing for arbitrarily small non-zero $m$. In figs. $1 \mathrm{~b}$ and $\mathrm{c}, M$ remains zero up to a critical value of $m$. The composite mass "turns on" only when the quark mass exceeds the critical value, continuously in fig. $1 \mathrm{~b}$, discontinuously in fig. 1c. (Since the only mass scale in QCD other than $m$ is the confinement scale $\Lambda$, we expect, if QCD actually behaves this way, that the critical quark mass is of order $A$.) 
a)

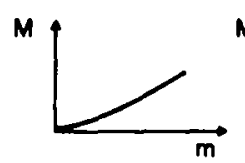

b)

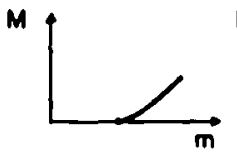

c)

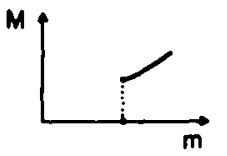

Fig. 1. Three possible types of behavior of the composite particle mass $M$ as a function of the intrinsic constituent mass $m$.

In this context, the PMC is the requirement that the behavior sketched in figs. $1 \mathrm{~b}$ and $\mathrm{c}$ cannot occur; that $M=0$ only if $m=0$ [11]. More generally, the PMC requires that a composite particle cannot stay massless while the mass of one of its constituents varies continuously over a range of values.

What is the motivation for this assumption? One might argue that it seems implausible that the dynamics responsible for binding a massless compdsite can adjust to keep the composite massless as the mass of a constituent varies. But this argument is not very persuasive. The chief motivation for the use of the PMC in refs. $[9,11]$ is that it is not unreasonable and has interesting consequences.

The PMC can be used to reach conclusions about how chiral symmetry is realized in two ways; the massless particles to which it is applied can be either spin $-\frac{1}{2}$ fermions or spin-0 Goldstone bosons ${ }^{\star}$. The application to spin- $-\frac{1}{2}$ fermions is exemplified by ref. [9]. Suppose that the unbroken subgroup $H_{f}$ of a flavor group $G_{f}$ requires that some multiplets of composite fermions be massless. We can deduce an algebraic constraint on the $\mathrm{H}_{\mathrm{f}}$ representation content of the massless fermions as follows: Imagine that some of the elementary fermions receive intrinsic bare mass which leave a subgroup $G_{f}^{\prime}<G_{f}$ as the exact flavor symmetry. If these intrinsic masses are very small, the unbroken exact flavor symmetry will be ${ }^{\star \star} G_{i}^{\prime} \cap H_{f}$. The fermion multiplets kept massless by $\mathrm{H}_{\mathrm{f}}$ symmetry can be decomposed into $\mathrm{G}_{\mathrm{f}}^{\prime} \cap \mathrm{H}_{\mathrm{f}}$ multiplets. The PMC requires that all composite fermions which contain massive constituents can acquire masses invariant under $\mathrm{G}_{f}^{\prime} \cap \mathrm{H}_{\mathrm{f}}$.

In ref. [9], 't Hooft found that this requirement could not be simultaneously satisfied with the anomaly condition by any set of massless composite fermion multiplets for $\mathrm{H}_{\mathrm{f}}=\mathrm{G}_{\mathrm{f}}=\mathrm{SU}(n)_{\mathrm{L}} \times \mathrm{SU}(n)_{\mathrm{R}} \times \mathrm{U}(1)_{\mathrm{V}}$ in $n$-flavor $\mathrm{QCD}$, with $n>2$. (He used $\mathrm{G}_{f}^{\prime}=\mathrm{SU}(n-1)_{\mathrm{L}} \times \mathrm{SU}(n-1)_{\mathrm{R}} \times \mathrm{U}(1)_{V} \times \mathrm{U}(1)_{V}^{\prime}$, the subgroup of $\mathrm{G}_{\mathrm{f}}$ which survives when one quark is massive.) Therefore, he concluded that $G_{f}$ must be spontaneously broken.

The PMC can also be applied to composite bosons, as follows [11]: Suppose a subgroup $S_{f} \subset G_{f}$ is non-chiral; that is, allows intrinsic masses for all the elementary fermions transforming non-trivially under $S_{f}$. (An example is $S_{f}=S U(n)_{v} \times U(1)_{V}$

\footnotetext{
* That these are the only possibilities is shown in ref. [14].

** To determine the intersection of $G_{f}^{\prime}$ and $H_{f}$, it is necessary to solve the subgroup alignment problem [12].
} 
in QCD.) Then a consequence of the PMC is that $\mathrm{S}_{\mathrm{f}}$ cannot be spontaneously broken. For if $S_{f}$ is spontaneously broken, there is an exactly massless Goldstone boson which is a bound state of elementary fermions transforming non-trivially under $S_{f}$. If the elementary fermions receive small $S_{f}$ invariant intrinsic masses, this Goldstone boson remains exactly massless; as a function of the intrinsic mass $m$, the Goldstone boson mass $M$ must behave as in fig. $1 \mathrm{~b}$ or $1 \mathrm{c}$, contradicting the PMC. Therefore, the PMC implies that non-chiral flavor symmetries cannot be spontaneously broken.

Combining the conclusions of refs. $[9,11]$ we know that the unbroken flavor symmetry group $\mathrm{H}_{f}$ of $n$-flavor QCD must be a proper (for $n>2$ ) subgroup of $\mathrm{G}_{f}=\mathrm{SU}(n)_{\mathrm{L}} \times \mathrm{SU}(n)_{\mathrm{R}} \times \mathrm{U}(1)_{\mathrm{V}}$ which contains $\mathrm{S}_{\mathrm{f}}=\mathrm{SU}(n)_{\mathrm{V}} \times \mathrm{U}(1)_{\mathrm{V}}$. The only possibility is $H_{f}=S_{f}$. Hence, assuming the PMC, we can prove that the flavor symmetry of $n$-flavor QCD must be spontaneously broken to $\mathrm{SU}(n)_{\mathrm{V}} \times \mathrm{U}(1)_{\mathrm{V}}$, if $n>2$. (For $n=2$, we cannot exclude the possibility that $S U(2)_{L} \times S U(2)_{R} \times U(1)_{V}$ is completely unbroken.)

We should note that, although applying the PMC yields a lot of information about how flavor symmetry is realized in QCD, it can be applied only to theories in which gauge-invariant mass terms are allowed for some of the elementary fermions. Perhaps the most fascinating gauge theories are those with non-real fermion representation content in which no gauge-invariant fermion masses are possible [5-8]. The PMC can tell us nothing about the realization of flavor symmetry in such a theory.

In the rest of this paper, we will show that it is possible to construct models in which the PMC is not valid, models in which massless composite particles contain massive constituents. Our examples are entertaining, and support the contention in ref. [11] that the PMC is not generally true. However, we cannot answer the question which originally motivated this work; we do not know if the PMC is valid in QCD.

\section{Vacuum alignment and Goldstone bosons}

The crucial feature of the models described in sects. 4 and 5 is that an intrinsic elementary fermion mass fails to align with a pattern of dynamical symmetry breaking. In this section, we present a simpler example which illustrates this idea ${ }^{*}$. The analysis of this example depends critically on the sign of a strong-interaction parameter which we cannot determine. Hence, we will proceed in sects. 4 and 5 to construct examples free of such ambiguities.

We consider two-flavor $Q C D$, and suppose that when the $u$ and $d$ quarks are exactly massless, the $S U(2)_{L} \times S U(2)_{R} \times U(1)_{V}$ chiral symmetry is spontaneously

\footnotetext{
* This example was suggested by Snyderman [19]. We thank him and Sidney Coleman for a helpful discussion concerning it.
} 
broken to $S U(2)_{V} \times U(1)_{v}$. This pattern of symmetry breaking is specified by a bilinear fermion condensate, which, in an appropriate basis, has the form

$$
\Phi=\left\langle\left(\begin{array}{cc}
\bar{u}_{\mathrm{L}} u_{\mathrm{R}} & \bar{u}_{\mathrm{L}} d_{\mathrm{R}} \\
\bar{d}_{\mathrm{L}} u_{\mathrm{R}} & \bar{d}_{\mathrm{L}} d_{\mathrm{R}}
\end{array}\right)\right\rangle \propto\left(\begin{array}{ll}
1 & 0 \\
0 & 1
\end{array}\right) .
$$

We might naively attempt to construct a theory with massive quarks and a massless Goldstone boson as follows: We introduce quark masses of the form

$$
\mathscr{H}=m\left(\bar{u}_{\mathrm{L}} d_{\mathrm{R}}-\bar{d}_{\mathrm{L}} u_{\mathrm{R}}\right)+\text { h.c. . }
$$

Equivalently, in the basis in which the quark mass matrix is diagonal, the condensate is

$$
\Phi \propto\left(\begin{array}{rr}
0 & 1 \\
-1 & 0
\end{array}\right)
$$

But flavor-diagonal quark masses leave u-number and d-number separately conserved. In particular, $u$-number minus d-number is an exact symmetry which is spontaneously broken by this condensate. Hence, there must be an exactly massless Goldstone boson which is a bound state of massive $u$ and $d$ quarks.

This construction actually fails for an obvious reason which becomes particularly clear if we think about an analogous ferromagnetic model [12]. If a spontaneous magnetization appears in an $\mathrm{O}(3)$-symmetric magnet along the $z$-axis,

$$
\langle\boldsymbol{M}\rangle=\mathrm{Me}_{z},
$$

then $\mathrm{O}(3)$ is spontaneously broken to $\mathrm{U}(1)$, and there are massless spin waves. Now suppose we turn on an external magnetic field along the $x$-axis, generating an $\mathrm{O}(3)$-breaking perturbation.

$$
H^{\prime}=B M_{x} .
$$

In the presence of this perturbation, the exact symmetry of the magnet consists of rotations about the $x$-axis, and this symmetry is spontaneously broken by a magnetization along the $z$-axis. Thus, we might still expect a massless spin wave.

We have, however, neglected the fact that it is energetically favorable for the magnetization to align with the external field. When it does so, the $U(1)$ left invariant by the magnetization coincides with the exact $U(1)$ symmetry, so there is no spontaneously broken exact symmetry, and no massless spin wave.

Similarly, both the condensate and the intrinsic masses in our QCD example leave unbroken an $S U(2) \times U(1)$ group. We should expect these two groups to align, so that there are no exactly massless Goldstone bosons [12].

Returning to the ferromagnet example, though, we note that it need not be the case that the symmetry groups of the perturbation and of the magnetization align. For suppose the perturbation actually has the form

$$
H^{\prime}=\left(B M_{x}\right)^{2} \text {, }
$$


which is also invariant under rotations about the $x$-axis. It is now energetically favorable for the magnetization to be perpendicular to the $x$-axis. Because the perturbation and the magnetization fail to align, there is a spontaneously broken exact $U(1)$ symmetry, and a massless spin wave, even in the presence of the perturbation.

Our task is to generalize this idea to relativistic quantum field theory. It is possible for the intrinsic quark masses and the quark condensate to fail to align in QCD, so that there is an exactly massless composite Goldstone boson with massive constituents?

The orientation of the quark condensate $\Phi$ in QCD is chosen to minimize the vacuum energy; to determine the correct orientation we must compute the minimum of an effective potential $V(\Phi)[12]$. When the quarks are exactly massless, this potential must be $S U(2)_{\mathrm{L}} \times S U(2)_{R} \times U(1)_{V}$ invariant. We will assume that the vacuum angle $\theta$ of QCD is either 0 or $\pi$, so that the potential is also invariant under $P$ and $C P[15]$.

The condensate can be expressed as

$$
\Phi=\pi_{0}+i \pi \cdot \tau+i \phi_{0}+\phi \cdot \tau,
$$

where $\tau$ is a $2 \times 2$ isospin matrix. The scalar fields $\pi$ and $\phi$ are 4 -vectors under $\mathrm{O}(4)=\mathrm{SU}(2)_{\mathrm{L}} \times \mathrm{SU}(2)_{\mathrm{R}}$; they can be construed as the degrees of freedom of a low-energy phenomenological field theory [16].

If, in the massless-quark limit, chiral $\mathrm{SU}(2)_{L} \times \mathrm{SU}(2)_{R}=\mathrm{O}(4)$ is spontaneously broken to $\mathrm{SU}(2)_{\mathrm{V}}=\mathrm{O}(3)$, and $P$ and $C P$ are not spontaneously broken, then either $\pi$ or $\phi$, but not both, is non-zero. (The $\mathrm{U}(1)_{\mathrm{A}}$ transformation which rotates $\pi$ into $\phi$ is not a symmetry of QCD, because of the axial anomaly [15].) We assume without loss of generality that $\pi$ is non-zero, and, performing an $\mathrm{O}(4)$ rotation, we are free to choose

$$
\pi_{0} \neq 0, \quad \pi=0 .
$$

Then $\pi$ represents the Goldstone boson triplet in the phenomenological field theory.

Now suppose we introduce a small chiral-symmetry-breaking quark mass term of the form

$$
\mathscr{H}^{\prime}=m\left(\bar{u}_{\mathrm{L}} u_{\mathrm{R}}-\bar{d}_{\mathrm{L}} d_{\mathrm{R}}\right) .
$$

Treated in lowest order perturbation theory, this perturbation will force $\phi$ to be non-zero and aligned with the quark mass matrix. Will $\pi$ also align with the quark mass matrix? If we (arbitrarily) truncate the potential by ignoring terms of higher than quartic order, then the only term invariant under $\mathrm{O}(4)$ and $C P$ which depends on the relative orientation of $\phi$ and $\pi$ is $(\phi \cdot \pi)^{2}$. To lowest order in the quark mass $m$, the potential has the form

$$
V(\phi, \pi)=A(\phi \cdot \pi)^{2}+m \phi_{3}+f\left(\phi^{2}, \pi^{3}\right),
$$




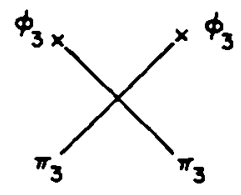

Fig. 2. Graph contributing to the mass-squared of the pseudo-Goldstone boson $\pi_{3}$.

where $f$ is minimized at $\phi^{2}=0, \pi^{2} \neq 0$. The minimum of $V$ occurs for $\phi_{3}=O(m)$; whether $\phi$ and $\pi$ align depends on the sign of $A$. If $A$ is positive; $V$ is minimized when $\phi$ and $\pi$ are orthogonal $O(4)$ vectors.

Therefore, if $\boldsymbol{A}$ is positive, the SU(2) isospin symmetry left invariant by the perturbation is spontaneously broken to $U(1)$, and $\pi_{1}$ and $\pi_{2}$ are exact Goldstone bosons with massive constituents. But $\pi_{3}$ is a pseudo-Goldstone boson which acquires a mass squared of order $m^{2}$ from fig. 2. (Notice that $C P$ is spontaneously broken whatever the sign of $\boldsymbol{A}$; this is an example of Dashen's phenomenon [12].)

The precise form of the potential in eq. (3.10) is not an essential feature of this example. The point is only that the potential will fix the relative orientation of $\pi$ and $\phi$; either they align or they do not, and if they do not, isospin is spontaneously broken. Unfortunately, we do not know how to calculate this potential so we cannot say whether isospin can be spontaneously broken in QCD as we have described here. In sect. 4 we will construct a similar example in which the potential which determines the alignment of the condensate is explicitly computable.

\section{A composite Goldstone boson with massive constituents}

The analysis of the models we construct in this section and in sect. 5 follows the pattern established in sect. 3. We will assume a realization of flavor symmetry in the flavor-symmetry limit; then we will consider the influence of flavor-symmetrybreaking perturbations on the orientation of a condensate. The perturbations will be a small fermion bare mass term as before, and also a weak gauge interaction which gauges a $U(1)_{W}$ subgroup of the flavor group. We will see that, if the $U(1)_{W}$ is chosen appropriately, the weak gauge interaction and fermion bare mass try to align the condensate in different directions. If the gauge interaction wins this competition, the condensate fails to align with the bare mass term, and there is a massless Goldstone boson with massive constituents, as in sect. 3 .

Our first example is a variant of two-flavor $Q C D$. The gauge group is $G_{S} \times U(1)_{w}$, where the $G_{S}$ interaction is a strong interaction with confinement scale $A$, and the $\mathrm{U}(1)_{W}$ interaction is a weak interaction which can be treated perturbatively. The fermions in this model are

$$
\mathbf{u}_{\mathrm{L}}^{0}, \quad \overline{\mathbf{u}}_{\mathrm{R}}^{0}, \quad \mathrm{~d}_{\mathrm{L},}^{1}, \quad \mathrm{~d}_{\mathrm{R}}^{-1}, \quad \mathbf{R},
$$

where $u, d$ are in equivalent complex irreducible representations of $G_{S}$, and have 
the $U(1)_{\mathrm{W}}$ charge assignments shown. $\mathrm{R}$ stands for a real reducible representation of $G_{s}$ with $U(1)_{W}$ charge assignments chosen so that $G_{s} G_{s} U(1)_{W}$ and $U(1)_{W}^{3}$ anomalies cancel. $R$ has been introduced so that we can gauge the $U(1)_{A}$ symmetry of the $d$ without encountering anomalies. We will suppose that $R$ self-condenses, spontaneously breaking $U(1)_{w}$. But because the $R$ condensate does not contribute to the effective potential we will minimize, it plays no essential role in the following discussion.

The $u$ has an explicit mass,

$$
\mathscr{H}_{\mathrm{m}}=m \bar{u}_{\mathrm{L}} u_{\mathrm{R}}+\text { h.c. },
$$

but in the limit in which $m$ and the $\mathrm{U}(1)$ gauge coupling $e$ vanish, this theory respects the global flavor symmetry

$$
\mathrm{G}_{\mathrm{f}}=\mathrm{SU}(2)_{\mathrm{L}} \times \mathrm{SU}(2)_{\mathrm{R}} \times \mathrm{U}(1)_{\mathrm{V}} \times \mathrm{U}(1)_{\mathrm{A}} .
$$

We will assume that $G_{f}$ is spontaneously broken by the strong $G_{s}$ interaction to the subgroup

$$
\mathrm{H}_{\mathrm{f}}=\mathrm{SU}(2)_{\mathrm{V}} \times \mathrm{U}(1)_{\mathrm{V}} \text {. }
$$

The orientation of $\mathrm{H}_{\mathrm{q}}$ in $\mathrm{G}_{\mathrm{f}}$ can be specified by an $\mathrm{H}_{\mathrm{f}}$-invariant fermion condensate. In the limit of exact $G_{f}$ symmetry, the orientation of the condensate is arbitrary, but when $m$ and $e$ are non-zero, the correct orientation is chosen to minimize the vacuum energy. We can compute and minimize an effective potential to determine how the condensate lines up $[1,12,17]$.

The orientation of the $\mathrm{H}_{\mathrm{f}}$-invariant condensate is labeled by four parameters, but it is shown in appendix $A$ that the effective potential can be expressed as a function of the single angle $\theta$ which defines the linear combination of $u_{\mathrm{R}}$ and $d_{\mathrm{R}}$,

$$
u_{\mathrm{R}}^{\prime}(\theta)=u_{\mathrm{R}} \cos \theta+d_{\mathrm{R}} \sin \theta,
$$

that condenses with $\bar{u}_{\mathrm{L}}$. Taking $m$ and $e^{2}$ to be small, we will calculate the effective potential to lowest order in $m$ and $e^{2}$. The contribution of order $m$ is easily seen to be

$$
V_{\mathrm{m}}(\theta)=\left\langle\mathscr{H}_{\mathrm{m}}\right\rangle=2 m \operatorname{Re}\left\langle\bar{u}_{\mathrm{L}} u_{\mathrm{R}}\right\rangle=-2 m \Delta_{\mathrm{m}} \cos \theta,
$$

where $\Delta_{m}$ is of order $\Lambda^{3}$ and can be chosen to be positive. $V_{m}$ is minimized at $\cos \theta=1$. The intrinsic $u$ mass encourages $\overline{\mathbf{u}}_{\mathrm{L}}$ to condense with $\mathrm{u}_{\mathrm{R}}$.

The contribution to the effective potential of order $e^{2}$ is computed in appendix A using the methods of ref. [17]. It has the form

$$
V_{\mathrm{W}}(\theta)=\frac{e^{2}}{16 \pi^{2}} \Delta_{\mathrm{w}} \cos ^{2} \theta
$$

where $\Delta_{\mathrm{W}}=\mathrm{O}\left(\Lambda^{4}\right)$ is expected to be positive $V_{\mathrm{W}}$ is minimized when $\cos \theta=0$.

* If $\Delta_{W}$ is actually negative, we can simply change the $U(1)_{W}$ charge assignments to reach the same conclusions as below. 
The $U(1)_{W}$ interaction tries to prevent the doubly charged fermion bilinear $\bar{d}_{L} d_{R}$ from condensing.

The two perturbations, the intrinsic u mass and the weak gauge interaction, compete with each other; which one wins will depend on the relative strength of $m$ and $e^{2} A$. If $m$ is sufficiently large, the potential

$$
V(\theta)=-2 m \Delta_{\mathrm{m}} \cos \theta+\frac{e^{2}}{16 \pi^{2}} \Delta_{\mathrm{w}} \cos ^{2} \theta
$$

is minimized at $\cos \theta=1$. But if $m$ is smaller than the critical value

$$
m_{\mathrm{c}}=\frac{e^{2}}{16 \pi^{2}} \frac{\Delta_{\mathrm{W}}}{\Delta_{\mathrm{m}}} \sim \frac{e^{2}}{16 \pi^{2}} \Lambda,
$$

a non-trivial minimum occurs at

$$
\cos \theta=\left(m \Delta_{m}\right) /\left(\frac{e^{2}}{16 \pi^{2}} \Delta_{W}\right)=\frac{m}{m_{\mathrm{c}}} .
$$

For $m<m_{\mathrm{c}}$, the $\mathrm{U}(1)_{\mathrm{W}}$ interaction is strong enough to prevent the condensate from aligning with the intrinsic $u$ mass.

This model has the exact global symmetry u-number minus d-number. When $\cos ^{2} \theta \neq 1$, this symmetry is spontaneously broken by the condensate $\left\langle\bar{u}_{\mathrm{L}} d_{\mathrm{R}}\right\rangle \neq 0$, and there is an exactly massless Goldstone boson coupling to the current

$$
J^{\mu}=\bar{q} \tau^{1} \gamma^{\mu} \gamma^{5} q \cos \theta+\bar{q} \tau^{3} \gamma^{\mu} q \sin \theta,
$$

where $q$ represents the $(u, d)$ doublet. The Goldstone boson is a massless composite particle that contains the massive u-quark.

The Applequist-Carazzone decoupling theorem [13] requires this composite Goldstone boson to become massive when $m$ is sufficiently large, which is exactly what we have found. When $m>m_{\mathrm{c}}$, the minimum of the potential is $\cos \theta=1$; the spontaneously broken global U(1) symmetry becomes restored, and the massless Goldstone boson becomes a massive pseudo-Goldstone boson. The pseudo-Goldstone boson mass $M$ is given by [17]

$$
M^{2}=\frac{4}{F^{2}}\left(m \Delta_{\mathrm{m}}-\frac{e^{2}}{16 \pi^{2}} \Delta_{\mathrm{W}}\right)=\frac{4}{F^{2}}\left(m-m_{\mathrm{c}}\right) \Delta_{\mathrm{m}} .
$$

The composite mass $M$ turns on continuously, as in fig. 1b. For $m<m_{\mathrm{c}}$, the Goldstone boson is a tachyon, signalling the instability of the vacuum in which u-number minus d-number is conserved.

We have found a composite Goldstone boson which remains exactly massless while one of its constituents receives an intrinsic mass, but only by assuming that $\mathrm{G}_{f}$ is spontaneously broken to $\mathrm{H}_{f}$ by the strong interaction. However, our model can easily be generalized to the $n$-flavor case, and we have seen in sect. 2 that 
confinement and the PMC imply that the $\mathrm{SU}(n)_{\mathrm{L}} \times \mathrm{SU}(n)_{\mathrm{R}} \times \mathrm{U}(1)_{V}$ chiral symmetry of $\mathrm{QCD}$ is spontaneously broken to $\mathrm{SU}(n)_{\mathrm{V}} \times \mathrm{U}(1)_{\mathrm{V}}$. Including the $\mathrm{R}$-fermions does not alter this conclusion. Therefore, assuming only confinement, we have proved the existence of a renormalizable quantum field theory in which the PMC is not satisfied.

\section{A massless composite fermion with a massive constituent}

Now we will apply the ideas of sect. 4 to a model with a massless composite fermion. The analysis of this model is based on more speculative assumptions than the analysis of the model in sect. 4.

The gauge group of the model is $\mathrm{SU}(5)_{\mathrm{S}} \times \mathrm{U}(1)_{\mathrm{W}}$, where $\mathrm{SU}(5)_{\mathrm{S}}$ is a strong interaction with confinement scale $\Lambda$, and $U(1)_{\mathrm{W}}$ is a weak interaction to be treated perturbatively. The fermions are in the SU(5) $\mathrm{S}$ representations

$$
10^{a}, 10^{b}, \quad \overline{10}^{-a}, \quad \overline{5}^{-3 b}, 1^{5 b} .
$$

All the fermions are left-handed, and the $U(1)_{W}$ charge assignments are as indicated. Note that this model is anomaly-free. There is also an intrinsic mass term

$$
\mathscr{H}_{\mathrm{m}}=m \overline{10}^{-a} 10^{a}
$$

allowed by the gauge symmetry.

Before proceeding with the analysis of this model, we review the hypothetical behavior of a simpler SU(5) model. Suppose the only fermions are

$$
Y=\frac{10}{1} \frac{\overline{5}}{-3} \text {. }
$$

This model has a global $\mathrm{U}(1)_{Y}$ symmetry with assignments as shown. How is the $\mathrm{U}(1)_{Y}$ symmetry realized?

Georgi [18] has argued that it is quite plausible that $U(1)_{Y}$ escapes spontaneous breakdown. As emphasized in ref. [6], there are two complementary ways of describing how this model behaves. In one description, the "Higgs picture", a bilinear fermion condensate forms which breaks SU(5) down to SU(4). Condensation occurs in the channels

$$
10 \times 10 \rightarrow \overline{5}, \quad 10 \times \overline{5} \rightarrow 5,
$$

and the $\overline{5}$ and 5 condensates are presumed to line up to leave the same SU(4) unbroken. There is one uncondensed fermion, the SU(4) singlet contained in the $\overline{5}$. We can define an unbroken global $U(1)$ generator $Y^{\prime}$ by combining $Y$ with a broken $S U(5)$ generator. The uncondensed fermion carries $Y^{\prime}=-5$, and is kept massless by the unbroken $U(1)$.

In the complementary "confinement picture", the SU(5) interaction is considered to be exactly confining; $\mathrm{SU}(5)$ is not spontaneously broken. The massless fermion 
is construed as a bound state,

$$
10 \overline{5} \overline{5}, \quad Y=-5 \text {. }
$$

This massless composite fermion saturates 't Hooft's anomaly condition.

There appears to be no physical distinction between these two descriptions. Therefore, if the $U(1)_{Y}$ symmetry is not spontaneously broken, we may interpret the resulting massless fermion as a composite state.

Now we return to the $S U(5)_{s} \times U(1)_{w}$ model with fermion content specified in (5.1). In the limit $m=e=0$, the flavor group of this model is

$$
G_{f}=S U(2) \times U(1) \times U(1) \times U(1) \text {. }
$$

It seems plausible to us that the strong $\mathrm{SU}(5)_{\mathrm{S}}$ interaction spontaneously breaks $G_{f}$ down to the subgroup

$$
H_{f}=U(1) \times U(1) \times U(1)
$$

We expect the $\overline{10}$ to condense with an arbitrary linear combination of the 10 's, leaving the orthogonal combination of the 10 's to bind with the $\overline{5}$ and produce a massless composite fermion.

When $m$ and $e$ are non-zero, the model still has an exact global $U(1) \times U(1)$ symmetry which keeps the composite fermion exactly massless. But the $G_{f}$ breaking perturbations will now determine the linear combination of the 10's,

$$
10(\theta)=10^{a} \cos \theta+10^{b} \sin \theta,
$$

that condenses with the $\overline{10}^{-a}$. We will argue that, for $e^{2}$ sufficiently large, and an appropriate choice of $a$ and $b, \cos ^{2} \theta \neq 1$. Then the 10 which binds with the $\overline{5}$ contains a component of the massive $10^{a}$; the massless composite fermion has a massive constituent.

We determine $\theta$ by minimizing the effective potential. The contribution of order $m$ to the potential is again of the form

$$
V_{\mathrm{m}}(\theta)=-2 m \Delta_{\mathrm{m}} \cos \theta .
$$

The order $e^{2}$ contribution is computed in appendix $\mathrm{B}$, and is found to be of the form

$$
V_{\mathrm{W}}(\theta)=\frac{e^{2}}{16 \pi^{2}} \Lambda^{4}\left[(a-b)(A a+B b) \cos 2 \theta+(a-b)^{2} C \cos ^{2} 2 \theta\right]
$$

where $A, B$, and $C$ are numerical factors of order 1 , determined by the strong $\mathrm{SU}(5)_{\mathrm{S}}$ dynamics.

Unfortunately, the arguments of ref. [17] do not suffice to establish the signs of $A, B$, and $C$. However, we are free to choose the $\mathrm{U}(1)_{\mathrm{W}}$ charge assignments as we please. In particular, because there is no reason to expect $A=-B$, we should be 
able to choose

$$
(a-b)(A a+B b)>0, \quad|A a+B b| \gg|(a-b) C| .
$$

If $a$ and $b$ satisfy (5.11), then the second term in eq. (5.10) is negligible and the coefficient of $\cos 2 \theta$ is positive.

Hence if (5.11) is satisfied, the potential has the same form as eq. (4.7). The critical value of the mass is

$$
m_{\mathrm{c}} \approx \frac{e^{2}}{16 \pi^{2}} \frac{A^{4}}{\Delta_{m}} 2(a-b)(A a+B b) \sim \frac{e^{2}}{16 \pi^{2}} \Lambda(a-b)(A a+B b) .
$$

For $m>m_{c}$, the minimum of the potential occurs at $\cos \theta=1$. The exact global $U(1) \times U(1)$ and the weakly gauged $U(1)_{W}$ symmetries are all unbroken. The massless composite fermion is a bound state of the massless $10^{b}$ and $5^{-3 b}$.

However, if $m<m_{c}$, the minimum is at $\cos ^{2} \theta \neq 1$. Because the fermion condensate fails to align with the intrinsic fermion mass, there is an exact Goldstone boson containing the massive $10^{a}$ and $\overline{10}^{-a}$. This Goldstone boson is eaten by the $U(1)_{w}$ gauge boson. An exact global $U(1) \times U(1)$ remains unbroken, and one of these U(1)'s enforces the masslessness of the composite fermion which contains the massive $10^{a}$.

As $m$ increases through $m_{\mathrm{c}}$, the $\mathrm{U}(1)_{\mathrm{W}}$ is restored; the eaten Goldstone boson smoothly becomes a pseudo-Goldstone boson, and the mass of the $U(1)_{W}$ gauge boson goes smoothly to zero. There is a massless composite fermion in both phases of the theory. But only for $m<m_{\mathrm{c}}$ does the massless composite fermion have a massive constituent.

Although we cannot calculate $A, B$, and $C$, we should observe that the phase transition in this model might be first order, for an appropriate choice of $U(1)_{W}$ charges $a$ and $b$. Provided

$$
C<0, \quad 0<\frac{A a+B b}{(a-b)(-C)}<5,
$$

the minimum of the potential $V(\theta)=V_{\mathrm{m}}(\theta)+V_{\mathrm{W}}(\theta)$ changes discontinuously at $m=m_{\mathrm{c}}$, as illustrated in fig. 3 .

In contrast with the examples cited in ref. [17], we have found that our weaklygauged $U(1)_{W}$ interaction sometimes prefers to align so that it is spontaneously broken, rather than unbroken. This result seems less counter-intuitive when we

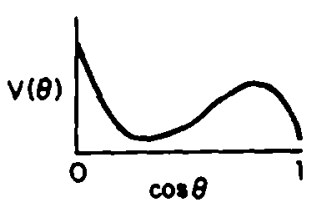

Fig. 3. A first-order phase transition. 
recall the "Higgs picture" for this model. The condensates (5.4) carry $U(1)_{W}$ charges [even when they are neutral under an appropriately redefined $U(1)_{w}^{\prime}$ ] so we should not be astonished if it turns out to be less costly energetically to break the $U(1)_{W}$ than to leave it unbroken.

Our demonstration that it is possible for a massless composite fermion to have a massive constituent has relied on the assumption that the global $U(1)_{Y}$ symmetry of the SU(5) model escapes spontaneous breakdown. There need be no massless fermion at all if $U(1)_{Y}$ is spontaneously broken. But our general strategy for constructing massless composite fermions with massive constituents can be carried out in almost any theory in which there are massless composite fermions when the elementary fermions are massless. Therefore, we can be reasonably confident that it is possible to construct models in which massless composite fermions have massive constituents, if massless composite fermions ever occur at all.

\section{Conclusions}

We have constructed models in which massless bosons or fermions are bound states of massive elementary fermions. The crucial ingredient in these models is a weak gauge interaction which prevents a fermion condensate from aligning with the intrinsic fermion masses.

In analyzing our models, we have made assumptions about how exact flavor symmetries are realized. But we have demonstrated that, given a confining gauge theory with a massless composite boson or fermion, it is often possible to modify the theory so that the composite remains massless while its constituents receive intrinsic masses.

In our example with a massless composite fermion, the requirement of decoupling is satisfied in an unusual way. As the intrinsic mass $m$ of the elementary fermion increases to infinity, a composite fermion remains massless. But at a critical value of $m$, the "wave function" of the composite changes, so that it no longer contains the massive elementary fermion. It would be more interesting if the unbroken symmetry actually decreased at the critical value of $m$, allowing the composite fermion to become massive. We do not know how to construct such an example.

We have shown that the persistent-mass condition is not generally true, but we have not shown that it is wrong in QCD, or in other asymptotically free gauge theories with a simple gauge group. One should note that the examples constructed in this paper and in ref. [11] require auxiliary dimensionless parameters to fix the critical value of the elementary fermion mass. In our examples this parameter is the $\mathrm{U}(1)_{\mathrm{w}}$ gauge coupling $e$, and the critical mass is $m_{\mathrm{c}} \sim\left(e^{2} / 16 \pi^{2}\right) A$. There is no dimensionless free parameter in QCD. It seems quite possible to us that the persistent-mass condition is correct for $\mathrm{QCD}$ and other zero-parameter quantum field theories. 
We thank Sidney Coleman, Estia Eichten, Neal Snyderman, and Steven Weinberg for valuable discussions, and we are also grateful for the hospitality of the Institute of Theoretical Physics at Santa Barbara.

\section{Appendix A}

Here we compute the order $e^{2} U(1)_{\mathrm{W}}$ contribution to the effective potential for the model described in sect. 4 .

The effective potential depends on the relative orientation of the two subgroups of $\mathrm{G}_{\mathrm{f}}=\mathrm{SU}(2)_{\mathrm{L}} \times \mathrm{SU}(2)_{\mathrm{R}} \times \mathrm{U}(1)_{\mathrm{V}} \times \mathrm{U}(1)_{A}$, the unbroken subgroup $\mathrm{H}_{\mathrm{f}}=$ $\mathrm{SU}(2)_{V} \times U(1)_{V}$ and the weakly gauged $U(1)_{W}$ subgroup. Because only the relative orientation matters, we may keep $H_{f}$ fixed and rotate only $U(1)_{w}$. The $U(1)_{w}$ current, which in a standard basis is

$$
\begin{aligned}
J_{\mathrm{W}}^{\mu}(\mathbb{0}, \mathbb{0}) & =\bar{d}_{\mathrm{L}} \gamma^{\mu} d_{\mathrm{L}}-\bar{d}_{\mathrm{R}} \gamma^{\mu} d_{\mathrm{R}}+(\mathrm{R} \text { piece }) \\
& =-\bar{q}_{\mathrm{L}} \gamma^{\mu} T^{3} q_{\mathrm{L}}+\bar{q}_{\mathrm{R}} \gamma^{\mu} T^{3} q_{\mathrm{R}}+\left(\mathrm{G}_{\mathrm{i}} \text { singlet }\right),
\end{aligned}
$$

becomes, under a $G_{f}$ rotation

$$
J_{\mathrm{W}}^{\mu}\left(U_{\mathrm{L}}, U_{\mathrm{R}}\right)=-\bar{q}_{\mathrm{L}} \gamma^{\mu} U_{\mathrm{L}}^{\dagger} T^{3} U_{\mathrm{L}} q_{\mathrm{L}}+\bar{q}_{\mathrm{R}} \gamma^{\mu} U_{\mathrm{R}}^{\dagger} T^{3} U_{\mathrm{R}} q_{\mathrm{R}}+\left(\mathrm{G}_{\mathrm{f}} \text { singlet }\right) .
$$

Here $q$ represents the $(u, d)$ doublet and $U_{\mathrm{L}}, U_{\mathrm{R}}$ are arbitrary unitary matrices.

The contribution to the vacuum energy arising from the exchange of one $U(1)_{W}$ gauge boson is

$$
V_{\mathrm{W}}\left(U_{\mathrm{L}}, U_{\mathrm{R}}\right)=-\frac{1}{2} i e^{2} \int \mathrm{d}^{4} x D_{\mu \nu}(x)\left\langle 0\left|\mathrm{~T}\left(J_{\mathrm{W}}^{\mu}(x) J_{\mathrm{W}}^{\nu}(0)\right)\right| 0\right\rangle .
$$

Because the vacuum $|0\rangle$ is $\mathrm{H}_{\mathrm{f}}$ invariant, we extract the $\mathrm{H}_{\mathrm{f}}$-invariant piece of the product of two $\mathrm{U}(1)_{\mathrm{W}}$ currents to obtain [17]

$$
\begin{aligned}
V_{\mathrm{W}}(U) & =4 e^{2} \operatorname{tr}\left(T^{3} U T^{3} U^{\dagger}\right) \frac{1}{2} i \int \mathrm{d}^{4} x D_{\mu \nu}(x)\langle 0| \mathrm{T}\left(J_{3 \mathrm{~L}}^{\mu}(x) J_{3 \mathrm{R}}^{\nu}(0)|0\rangle+\right.\text { constant } \\
& =\frac{e^{2}}{8 \pi^{2}} \Delta_{\mathrm{W}} \operatorname{tr}\left(T^{3} U T^{3} U^{\dagger}\right)+\text { constant }
\end{aligned}
$$

where $U=U_{\mathrm{l}}^{\dagger} U_{\mathrm{R}}$ and $\Delta_{\mathrm{w}}$ is the spectral integral

$$
\Delta_{\mathrm{W}}=\frac{3}{4} \int \mathrm{d} S \ln \left(\frac{S_{0}}{S}\right)\left[\rho_{\mathrm{V}}(S)-\rho_{\mathrm{A}}(S)\right]
$$

$\Delta_{\mathrm{W}}$ is of order $\Lambda^{4}$, and according to the arguments of ref. [17], it is reasonable to expect that $\Delta_{\mathrm{W}}$ is positive. 
The matrix $U$ is a $2 \times 2$ unitary matrix

$$
U=\mathrm{e}^{i \gamma}\left[\begin{array}{cc}
\mathrm{e}^{i \alpha} \cos \theta & -\mathrm{e}^{i \beta} \sin \theta \\
\mathrm{e}^{-i \beta} \sin \theta & \mathrm{e}^{-i \alpha} \cos \theta
\end{array}\right] .
$$

(If we had chosen to keep $U(1)_{W}$ fixed and had rotated $H_{f}$ instead, the fermion condensate which specifies $H_{f}$ would have been proportional to $U^{+}$.) From (A.4) and (A.6) we find

$$
V_{\mathrm{w}}(\theta)=\frac{e^{2}}{16 \pi^{2}} \Delta_{\mathrm{w}} \cos ^{2} \theta .
$$

$V_{\mathrm{w}}(\theta)$ is independent of $\alpha, \beta$, and $\gamma$ because, when $\cos ^{2} \theta \neq 1$, there are three spontaneously broken $U(1)$ symmetries which commute with $U(1)_{w}$.

In the model described in sect. 4 , the lowest-order contribution to the potential due to the mass of the $u$-quark is

$$
V_{\mathrm{m}}(\theta, \alpha+\gamma)=-2 m \Delta_{\mathrm{m}}^{\prime} \cos (\alpha+\gamma) \cos \theta,
$$

which is minimized for $\cos (\alpha+\gamma)= \pm 1$. We have used our freedom to choose the sign of $\cos (\alpha+\gamma)$ to make $\Delta_{\mathrm{m}}$ positive in eq. (4.6). The parameters $\alpha-\gamma$ and $\beta$ are arbitrary, and correspond to two exactly massless Goldstone bosons. One of these is eaten by the $\mathrm{U}(1)_{\mathrm{W}}$ gauge boson, the other is the Goldstone boson discussed in sect. 4.

\section{Appendix B}

Here we compute the order $e^{2} \mathrm{U}(1)_{\mathrm{W}}$ contribution to the effective potential for the model described in sect. 5 .

As in appendix $A$, we will keep fixed the orientation of $H_{f}=U(1) \times U(1) \times U(1)$ in $\mathrm{G}_{f}=\mathrm{SU}(2) \times \mathrm{U}(1) \times \mathrm{U}(1) \times \mathrm{U}(1)$, and rotate $\mathrm{U}(1)_{\mathrm{W}}$. In a standard basis, the $\mathrm{U}(1)_{\mathrm{W}}$ current is

$$
J_{\mathrm{W}}^{\mu}(\mathbb{J})=(a-b) \bar{q}_{\mathrm{L}} \gamma^{\mu} T^{3} q_{\mathrm{L}}+\left(\mathrm{G}_{\mathrm{f}} \text { singlet }\right)
$$

where $q_{\mathrm{L}}$ is the $\left(10^{a}, 10^{b}\right)_{\mathrm{L}}$ doublet. Under an $\mathrm{SU}(2) \subset \mathrm{G}_{\mathrm{f}}$ rotation specified by

$$
U=\left[\begin{array}{cc}
\mathrm{e}^{i \alpha} \cos \theta & -\mathrm{e}^{i \beta} \sin \theta \\
\mathrm{e}^{-i \beta} \sin \theta & \mathrm{e}^{-i \alpha} \cos \theta
\end{array}\right],
$$

$T^{3}$ becomes

$$
T^{3 \prime}=U^{\dagger} T^{3} U=T^{3} \cos 2 \theta-\sin 2 \theta\left[T^{1} \cos (\alpha-\beta)+T^{2} \sin (\alpha-\beta)\right] .
$$

Again the effective potential in order $e^{2}$ is given by (A.3), and we must extract the $\mathrm{H}_{\mathrm{f}}$-invariant piece of the product of two $\mathrm{U}(1)_{\mathrm{W}}$ currents. $T^{1}$ and $T^{2}$ are an $\mathrm{H}_{\mathrm{f}}$ doublet, but $\mathrm{H}_{t}$ symmetry cannot prevent the $T^{3}$ component of $J_{\mathrm{W}}^{\mu}$ from mixing 
with the $G_{f}$-singlet component. Therefore, the most general form of the effective potential consistent with $\mathrm{H}_{\mathrm{f}}$ symmetry is

$V_{\mathrm{w}}(\theta)=\frac{e^{2}}{16 \pi^{2}} \Lambda^{4}\left[(a-b)^{2} C \cos ^{2} 2 \theta+(a-b)(A a+B b) \cos 2 \theta\right]+$ constant,

where $A, B$, and $C$ are numbers of order one determined by the strong $\mathrm{SU}(5)_{\mathrm{S}}$ dynamics. $V_{\mathrm{W}}(\theta)$ is a function of the single angle $\theta$ because, for $\cos ^{2} \theta \neq 1$, there are two spontaneously broken $U(1)$ symmetries which commute with $U(1)_{W}$.

Because of the uncontrolled mixing of the $G_{f}$-triplet and $G_{f}$-singlet currents, the arguments of ref. [17] do not suffice to determine the signs of $A, B$, and $C$.

\section{References}

[1] S. Weinberg, Phys. Rev. D13 (1976) 974; D19 (1979) 1277

[2] L. Susskind, Phys. Rev. D20 (1979) 2619

[3] S. Dimopoulos and L. Susskind, Nucl. Phys. B155 (1979) 237

[4] E. Eichten and K. Lane, Phys. Lett. 99B (1980) 125

[5] S. Dimopoulos, S. Raby and L. Susskind, Nucl. Phys. B169 (1980) 373

[6] S. Dimopoulos, S. Raby and L. Susskind, Nucl. Phys. B173 (1980) 208

[7] M.E. Peskin, Cornell preprint CLNS 81/503 (1981)

[8] S. Dimopoulos and L. Susskind, Nucl. Phys. B191 (1981) 370

[9] G. 't Hooft, in Recent developments in gauge theories, ed. G. 't Hooft et al. (Plenum Press, New York, 1980)

[10] Y. Frishman, A. Schwimmer, T. Banks and S. Yankielowicz, Nucl. Phys. B177 (1981) 157;

S. Coleman and B. Grossman, to be published

[11] J. Preskill and S. Weinberg, Phys. Rev. D24 (1981) 1059;

I. Bars, Yale preprint YTP81-09

[12] R. Dashen, Phys. Rev. D3 (1971) 1879

[13] T. Appelquist and J. Carazzone, Phys. Rev. D11 (1975) 2856

[14] S. Weinberg and E. Witten, Phys. Lett. 96B (1980) 59

[15] G. 't Hooft, Phys. Rev. Lett. 37 (1976) 8;

R. Jackiw and C. Rebbi, Phys. Rev. Lett. 37 (1976) 172;

C. Callan, R. Dashen and D. Gross, Phys. Lett. 63B (1976) 334

[16] P. Sikivie, L. Susskind, M. Voloshin and V. Zakharov, Nucl. Phys. B173 (1980) 189;

N. Snyderman, SLAC-PUB-2636 (1980)

[17] M.E. Peskin, Nucl. Phys. B175 (1980) 197;

J. Preskill, Nucl. Phys. B177 (1981) 21

[18] H. Georgi, Nucl. Phys. B156 (1979) 126

[19] N. Snyderman, unpublished 